impact of uncertainty, the response to colleague's clinical error and strategies to address uncertainty. Data obtained included gender and year of graduation. A likert scoring system was used (cuing at 1 , not at all and at 6, definitely), to rate responses. A don't know option was also allowed.

Results Forty two (100\%) trainees responded. The M:F was 1:2.9. The mean Likert scores for factors impacting on clinical decisions were 5.7 for clinical knowledge and experience, 5.4 for opinion of senior colleagues, 4.6 for fear of making a mistake, 4.2 for parent anxieties and expectations. Twenty one (50\%) of trainees frequently reflect on cases when at home. Their confident decisions are modified by opinions of senior colleague mean likert score 4. Mean Likert scores for responses to error scenario by a colleague were sympathy 4.3, acceptance 4.4, and anxiety 3. Mean Likert scores for strategies to address uncertainty included enhanced teaching 5.9, enhanced clinical experience 5.8, knowledge of 'red flags' 5.7, mentoring 5.6, building resilience 4.9, and developing self awareness 4.5.

Conclusion Paediatric trainees are impacted by clinical uncertainty which transfers into their home life. Incorporating strategies in training to enhance coping skills is necessary.

\section{SIMBA STUDY: UNDERGRADUATE MODULE IN SIMULATION TRAINING AND BASIC LIFE SUPPORT}

doi:10.1136/archdischild-2012-302724.1010

${ }^{1} \mathrm{~F}$ McElligott, ${ }^{2} \mathrm{H}$ Bruell, ${ }^{2} \mathrm{~S}$ Viash, ${ }^{2,3} \mathrm{~A}$ Nicholson, ${ }^{1,2,4}$ E Molloy. ${ }^{1}$ National Maternity Hospital, Holles Street; ${ }^{2}$ Royal College of Surgeons; ${ }^{3}$ Paediatrics, Children's University Hospital; ${ }^{4}$ University College Dublin, Dublin, Ireland

Background Medical education has been greatly enhanced by the development of simulation 'patients.' Real-time training with simulated clinical signs promotes development and institution of practical management plans, without the fear of causing harm. We aimed to evaluate confidence levels and personal preference, as well as overall satisfaction with the module.

Method Students received pre-course reading material and three teaching sessions, including didactic teaching, video and live demonstrations, basic life support training using mannequins and small group teaching. The final session was a thirty-minute session in groups of 6, in which the students worked in teams to assess, diagnose and treat a simulation baby, followed by a case discussion. Students completed anonymous questionnaires.

Results 164 students completed the first two questionnaires, and 140 students completed the third. There were 44 postgraduate entry students: 10 Masters \& $2 \mathrm{PhD}$ graduates. 131 students felt they had benefited from the pre-course reading material and 130 students felt the 2 -session pre-simulation were beneficial. Rated on a scale of $1-10$, students' confidence increased by 2.6 points after the simulation session. Following the simulation, 73 students planned to increase their clinical versus their book work. 76 students requested 2 or more further hours of simulation teaching and 121 found the simulation baby to be superior to mannequins as a teaching tool.

Discussion The introduction of the SIMBA module was well received amongst medical undergraduates. Students gave positive feed back. Technological advances of simulation patients greatly add to paediatric undergraduate training, and enhance overall experience.

\section{INTRODUCING VIDEO AS A SUPPLEMENT IN THE ASSESSMENT OF STUDENT PERFORMANCE IN CLINICAL PEDIATRIC EXAMINATION}

doi:10.1136/archdischild-2012-302724.1011

'M Malon, ${ }^{2} \mathrm{D}$ Cortes, ${ }^{1} \mathrm{G}$ Greisen, ${ }^{2} \mathrm{H}$ Simonsen, ${ }^{3} \mathrm{JL}$ Sørensen, ${ }^{4} \mathrm{~J}$ Andersen, ${ }^{5} \mathrm{U}$ Nygaard, ${ }^{5} \mathrm{~A}$ Poulsen, ${ }^{6} \mathrm{HB}$ Mortensen. 'Neonatal Clinic, Rigshospitalet, Copenhagen; ${ }^{2}$ Pediatric
Department, Hvidovre Hospital, Hvidovre; ${ }^{3}$ Department of Gynecology and Obstetrics, Rigshospitalet, Copenhagen; "Pediatric Department, Hillerød Hospital, Hillerød; ${ }^{5}$ Pediatric Department, Rigshospitalet, Copenhagen; ${ }^{6}$ Pediatric Department, Herlev Hospital, Herlev, Denmark

Background and Aims It is time consuming to include patients in the clinical pediatric examination of medical students. At Copenhagen University we perform a 25 min oral examination, based on a clinical paper case and wanted to evaluate supplemental exam questions, based on video

Methods With written parental consent 17 videos lasting $30 \mathrm{sec}-$ onds were recorded, demonstrating children with common clinical conditions. After the case-based examination, the student blindly chose one of the videos and was allowed to see the video twice, before assessing the specific symptoms and general appearance of child.

After the examination, the internal and external examiner answered a simple questionnaire.

Results 141 students were examined using videos. The videos were rated as suitable by $89 \%(92 / 103)$ of the internal examiners and by $76 \%(78 / 103)$ of the external examiners (Fishers Exact test, $\mathrm{p}<0.05)$

Overall, the internal and external examiners rated the video part on a 5-point Likert scale to be important or very important in $38 \%(40 / 106)$ and $36 \%(37 / 103)$ of the exams, respectively (Fishers Exact test, $\mathrm{p}=0.886)$. The internal examiners reported, that the video had changed the grades in $40 \%(42 / 106)$ of the exams, which was similar to $37 \%(38 / 103)$ for external examiners (Fishers Exact test, $p=0.776)$. Overall the grades at the examination were unchanged, median $B$ (range $G$ to $A$ ), $n=117$ before and median $B$ (range $\mathrm{F}$ to $\mathrm{A}$ ), $\mathrm{n}=141$ (Mann-Whitney test, $\mathrm{p}=0.992$ ) after videos were included.

Conclusions A short video showing a child with a common clinical condition appears to contribute significant to an oral examination based on a paper case.

\section{THE PERCEPTION AND IMPACT OF A 'BREAKING BAD NEWS' MODULE ON PAEDIATRIC MEDICAL STUDENTS}

doi:10.1136/archdischild-2012-302724.1012

'F McElligott, ${ }^{2,3} \mathrm{~A}$ Nicholson, ${ }^{1,3,4}$ EJ Molloy. ${ }^{1}$ National Maternity Hospital, Holles Street; ${ }^{2}$ Paediatrics, Children's University Hospital; ${ }^{3}$ Royal College of Surgeons; ${ }^{4}$ University College Dublin, Dublin, Ireland

Background Although, communication is an integral part of medical practice, it is also the source of $20-50 \%$ of patient complaints. Breaking bad news is a challenge across all disciplines yet little time is devoted to formal training and practice at any stage of medical training. We aimed to improve medical student confidence in delivering bad news in an appropriate and sensitive manner.

Methods Students received a teaching session including didactic teaching, discussions, video example and scenario group work. Students completed an anonymous questionnaire after the session, which assessed their basic demographics, aspects of breaking bad news (BBN) and their confidence in $\mathrm{BBN}$ pre and post the session.

Results 114 students completed the questionnaire, of whom 59 were male and 74 were undergraduate-entry. 110/114 had previously considered that breaking bad news might be an important part of their future jobs however only $73 / 112$ had previously considered that breaking bad news might consume a lot of time in their future jobs. 83 had witnessed professionals breaking bad news to either patients or family members in their training, and most commonly this was in paediatrics $(n=40)$, General Surgery $(n=24)$ and adult medicine $(n=45)$. The average change in confidence in breaking bad news was +2.46 (range -1 to 6 ). Students rated the session as $7.79 / 10$ for usefulness and 8.22 for interest. 